\title{
119.
}

\section{ON A THEOREM FOR THE DEVELOPMENT OF A FACTORIAL.}

[From the Philosophical Magazine, vol. vi. (1853), pp. 182-185.]

THE theorem to which I refer is remarkable for the extreme simplicity of its demonstration. Let it be required to expand the factorial $\overline{x-a} \overline{x-b} \overline{x-c} \ldots$ in the form

$$
\overline{x-\alpha} \overline{x-\beta} \overline{x-\gamma} \ldots+B \overline{x-\alpha} \overline{x-\beta} \ldots+C \overline{x-\alpha} \ldots+D \ldots \& \mathrm{c} .
$$

We have first

$$
x-a=\overline{x-\alpha}+\overline{\alpha-a}
$$

multiply the two sides of this by $\overline{x-b}$; but in multiplying by this factor the term $\overline{x-\alpha}$, write the factor in the form $\overline{x-\beta}+\overline{\beta-b}$; and in multiplying the term $\overline{\alpha-a}$, write the factor in the form $\overline{x-\alpha}+\overline{\alpha-b}$; the result is obviously

$$
\overline{x-a} \overline{x-b}=\overline{x-\alpha} \overline{x-\beta}+(\overline{x-a}+\overline{\beta-b}) \overline{x-\alpha}+\overline{\alpha-a} \overline{\alpha-b} ;
$$

multiply this by $x-c$, this factor being in multiplying the quantity on the right-hand side written successively under the forms $\overline{x-\gamma}+\overline{\gamma-c}, \overline{x-\beta}+\overline{\beta-c}, \overline{a-\alpha}+\overline{\alpha-c}$; the result is

$$
\begin{gathered}
\overline{x-a} \overline{x-b} \overline{x-c}= \\
+(\overline{\alpha-a}+\overline{\beta-b}+\overline{\gamma-c}) \overline{x-\alpha} \overline{x-\beta} \overline{x-\beta} \overline{x-\gamma} \\
+(\overline{\alpha-a} \overline{\alpha-b}+\overline{\alpha-a} \overline{\beta-c}+\overline{\beta-b} \overline{\beta-c}) \overline{x-\alpha} \\
+\overline{\alpha-a} \overline{\alpha-b} \overline{\alpha-c},
\end{gathered}
$$


which may be thus written,

$$
\begin{aligned}
& (x-a)(x-b)(x-c)= \\
& (x-\alpha)(x-\beta)(x-\gamma)+\left[\begin{array}{lll}
\alpha, & \beta, & \gamma \\
a, & b, & c
\end{array}\right]_{1} \overline{x-\alpha} \overline{x-\beta}+\left[\begin{array}{lll}
\alpha, & \beta, \\
a, & b, & c
\end{array}\right]_{2} \overline{x-\alpha}+\left[\begin{array}{lll}
\alpha & \\
a, & b, & c
\end{array}\right]_{3}
\end{aligned}
$$

Consider, for instance,

$$
\left[\begin{array}{lll}
a, & \beta, \\
a, & b, & c
\end{array}\right]_{2}=\overline{\alpha-a} \overline{\alpha-b}+\overline{\alpha-a} \overline{\beta-c}+\overline{\beta-b} \overline{\beta-c}
$$

then, paying attention in the first instance to the Greek letters only, it is clear that. the terms on the second side contain the combinations two and two, with repetitions, of the Greek letters $\alpha, \beta$, and these letters appear in each term in the alphabetical order. Each such combination may therefore be considered as derived from the primitive combination $\alpha, \alpha$ by a change of one or both of the $\alpha$ 's into $\beta$; and if we take (instead of the mere combination $\alpha, \alpha$ ) the complete first term $\overline{\alpha-a} \overline{\alpha-b}$, and simultaneously with the change of the $\alpha$ of either of the factors into $\beta$ make a similar change in the Latin letter of the factor, we derive from the first term the other terms of the expression on the right-hand side of the expression. It is proper also to remark, that, paying attention to the Latin letters only, the different terms contain all the combinations two and two, without repetitions, of the letters $a, b, c$. The same reasoning will show that

$$
\begin{aligned}
\overline{x-a} \overline{x-b} \overline{x-c} \overline{x-d}= & \overline{x-\alpha} \overline{x-\beta} \overline{x-\gamma} \overline{x-\delta} \\
& +\left[\begin{array}{llll}
\alpha, & \beta, & \gamma, & \delta \\
a, & b, & c, & d
\end{array}\right]_{1}^{\overline{x-\alpha} \overline{x-\beta} \overline{x-\gamma}} \\
& +\left[\begin{array}{llll}
\alpha, & \beta, & \gamma & \\
a, & b, & c, & d
\end{array}\right]_{2}^{\overline{x-\alpha} \overline{x-\beta}} \\
& +\left[\begin{array}{llll}
\alpha & \beta & \\
a, & b & c, & d
\end{array}\right]_{3}^{\overline{x-\alpha}} \\
& +\left[\begin{array}{llll}
\alpha & & \\
a, & b, & c, & d
\end{array}\right]_{4}
\end{aligned}
$$

where, for instance,

$$
\begin{aligned}
{\left[\begin{array}{llll}
\alpha, & \beta & & \\
a, & b, & c, & d
\end{array}\right]_{3}=} & (\alpha-a)(\alpha-b)(\alpha-c) \\
+ & (\alpha-a)(\alpha-b)(\beta-d) \\
+ & (\alpha-a)(\beta-c)(\beta-d) \\
+ & (\beta-b)(\beta-c)(\beta-d), \& c .
\end{aligned}
$$


It is of course easy, by the use of subscript letters and signs of summation, to present the preceding theorem under a more condensed form; thus writing

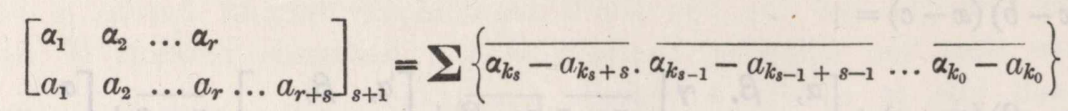

where $k_{s}, k_{s-1}, \ldots k_{0}$ form a decreasing series (equality of successive terms not excluded) of numbers out of the system $r, \overline{r-1}, \ldots 3,2,1$; the theorem may be written in the form

$$
\overline{x-a_{1}} \overline{x-a_{2}} \ldots \overline{x-a_{p}}=\boldsymbol{S}_{q^{0}}^{p}\left[\begin{array}{lllll}
\alpha_{1} & \alpha_{2} & \ldots & \alpha_{p-q+1} \\
a_{1} & a_{2} & \ldots & \ldots & a_{p}
\end{array}\right]_{q} \overline{x-\alpha_{1}} \overline{x-\alpha_{2}} \ldots \overline{x-\alpha_{p-q}}
$$

but I think that a more definite idea of the theorem is obtained through the notation first made use of. It is clear that the above theorem includes the binomial theorem for positive integers, the corresponding theorem for an ordinary factorial, and a variety of other theorems relating to combinations.

Thus, for instance, if $C_{q}\left(a_{1}, \ldots a_{p}\right)$ denote the combinations of $a_{1}, \ldots a_{p}, q$ and $q$ together without repetitions, and $H_{q}\left(a_{1}, \ldots a_{p}\right)$ denote the combinations of $a_{1}, \ldots a_{p}$, $q$ and $q$ together with repetitions, then making all the $\alpha$ 's vanish,

and therefore

$$
\overline{x-a}_{1} \ldots \overline{x-a_{p}}=S_{q^{0}}^{p}(-)^{q} C_{q}\left(a_{1}, \ldots a_{p}\right) x^{p-q}
$$

$$
(x-a)^{p}=\boldsymbol{S}_{q^{0}}^{p}(-)^{q} C_{q}(a, a \ldots \text { plures }) x^{p-q}=\boldsymbol{S}_{q^{0}}^{p}(-)^{q} \frac{[p]^{q}}{[q]^{q}} a^{q} x^{p-q},
$$

the ordinary binomial theorem for a positive and integral index $p$.

So making all the $a$ 's vanish,

$$
x^{p}=S_{q 0}^{p} H_{q}\left(\alpha_{1} \ldots \alpha_{p-q+1}\right) \overline{x-\alpha_{1}} \overline{x-\alpha_{2}} \ldots \overline{x-\alpha}_{p-q} .
$$

If $m$ be any integer less than $p$, the coefficient of $x^{m}$ on the right-hand side must vanish, that is, we must have identically

$$
0=\boldsymbol{S}_{q^{0}}^{p-m}(-)^{q} C_{p-q-m}\left(\alpha_{1}, \boldsymbol{\alpha}_{2}, \ldots \alpha_{p-q}\right) H_{q}\left(\alpha_{1}, \boldsymbol{\alpha}_{2}, \ldots \alpha_{p-q+1}\right) .
$$

So also

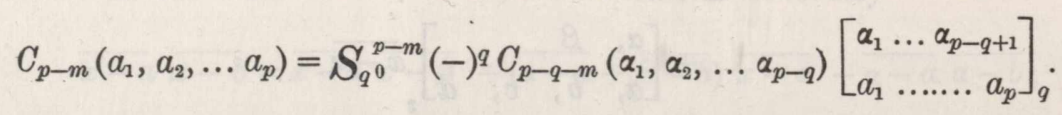

Suppose

$$
a_{1}=0, \quad a_{2}=1 \ldots a_{p}=p-1 ; \quad \alpha_{1}=k, \quad a_{2}=k-1, \ldots a_{p}=k-p+1,
$$

then

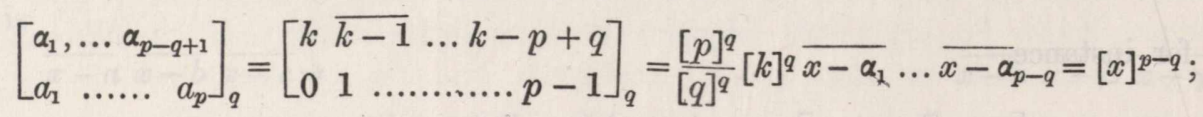

and hence

$$
[x+k]^{p}=\boldsymbol{S}_{q^{0}}^{p}[q]^{q}[q]^{q}[k]^{q}[x]^{p-q},
$$

the binomial theorem for factorials. 
A preceding formula gives at once the theorem

$$
H_{q}(0,1, \ldots p-q)=\frac{1}{[p-q]^{p-q}} \Delta^{p-q} 0^{p} .
$$

It may be as well to remark, with reference to a demonstration frequently given of the binomial theorem, that in whatever way the binomial theorem is demonstrated for integer positive indices, it follows from what has preceded that it is quite as easy to demonstrate the corresponding theorem for the factorial $[m]^{p}$. But the theorem being true for the factorial $[m]^{p}$, it is at once seen that the product of the series for $(1+x)^{m}$ and $(1+x)^{n}$ is identical with the series for $(1+x)^{m+n}$, and thus it becomes unnecessary to employ for the purpose of proving this identity the so-called principle of the permanence of equivalent forms; a principle which however, in the case in question, may legitimately be employed. 